\title{
Conservative Management of Thoracolumbar Spinal Tuberculosis in The Tertiary Care Hospital of India
}

\author{
Ghanshyam Kakadiya ${ }^{1}$, Yogesh Soni ${ }^{2}$, Kalpesh Saindane ${ }^{1}$, Kushal Gohil ${ }^{1}$, \\ Kshitij Chaudhary ${ }^{1,3}$, Akash Shakya ${ }^{1}$
}

\section{Abstract}

Introduction: Spinal tuberculosis is a leading cause of non-traumatic paraplegia in a developing country like India. There is an emerging trend to operate on patients early with spinal TB. A study aim was to reiterate the importance of conservative management in Spinal Tuberculosis. The study aim was to assess the clinical and radiological outcomes of the conservative management of thoracolumbar spine tuberculosis and reiterate the importance of conservative management.

Methods: A prospective study with 188 thoracolumbar tuberculosis patients included from May 2016 to April 2019. All the patients were subjected to computed tomography-guided biopsy followed by anti-tuberculous therapy (ATT) for 12-months. Indications for surgery included patients in which biopsy either failed and persistent/worsening of neurology. Preoperative and postoperative clinical and functional outcomes Visual Analog Scale (VAS), Oswestry Disability Index (ODI), and ASIA scale were measured.

Results: 160 patients had a neurological deficit of ASIA-C and ASIA-D in 28. A failed performed biopsy was in 18 patients. Out of 170 successful biopsies, 18 patients had persistent/worsening of neurology, these 18 (10.58\%) patients required surgery. VAS was significantly improved from mean value preoperative $7.90 \pm 0.60$ to $4.0 \pm 0.54$ postoperative 1 month and $2.90 \pm 0.54$ at the final follow-up. ODI was improved from mean value preoperative $77.10 \pm 6.90$ to $30.50 \pm 6.50$ postoperative 1 month and $21.30 \pm 6.70$ at final follow-up. Pre-treatment mean kyphosis was $5.68 \pm 3.84$ that improved to $6.51 \pm 3.88$ post-treatment.

Conclusions: The results of conservative treatment consisting of biopsy and ATT for at least 12 months in compliant patients are excellent. A combined approach using clinical staging, biopsy, and ATT can minimize surgical intervention in most patients. Early diagnosis and early treatment lead to a good prognosis. Periodic evaluation is a must to look for evidence of improvement and the adverse effect of ATT.

Keywords: Spinal tuberculosis; Biopsy; Conservative Management; ATT.

\section{Introduction}

Tuberculosis has been known to one of the first infectious diseases of mankind since the early civilizations. Tuberculosis has been mentioned in ancient Indian religious textbooks and termed yakima-wasting disease in the Rigveda $(1500 \mathrm{BC})$ and balasa in the Atharvaveda. The Sushruta Samhita (600BC) recommended treatment of it with breast milk, various meats, rest and fresh air [1]. The tuberculosis of the spine is popularly

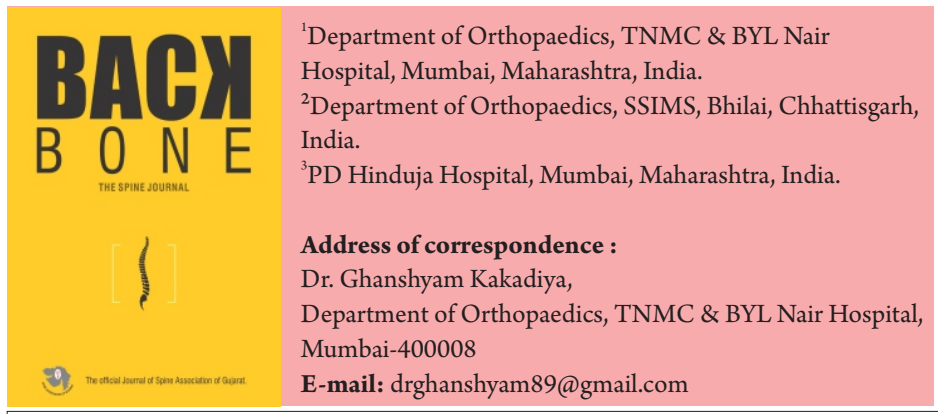

2021 (C) Back Bone: The Spine Journal (The Official Journal Of "Spine Association Of Gujarat") | Available on www.backbonejournal.com | DOI:10.13107/bbj.2021.v02i01.013 This is an Open Access article distributed under the terms of the Creative Commons Attribution Non-Commercial License (http://creativecommons.org/licenses/by-nc/3.0) which permits unrestricted non-commercial use, distribution, and reproduction in any medium, provided the original work is properly cited. known as Pott's spine. The name traces back its origin from the description of tuberculous infection of the spine by Sir Percival Pott [2]. Spinal tuberculosis is the most frequently encountered extra pulmonary form of tuberculosis and it accounts for approximately $50 \%$ of the cases of osteoarticular tuberculosis, and the thoracolumbar spine is the most common region within the spine to be affected [3]. Despite the widespread availability of antitubercular chemotherapy (ATT) and a better understanding of the natural history of the disease, tuberculosis remains an international health problem and the leading cause of mortality. The World Health Organization (WHO) Global Tuberculosis Report 2017 presents that tuberculosis is the ninth leading cause of death worldwide and the leading cause of a single infectious agent. About 10.4 million people were newly diagnosed to have tuberculosis worldwide in 2016, with 2.8 million cases being reported from India alone [4]. In developing countries like 

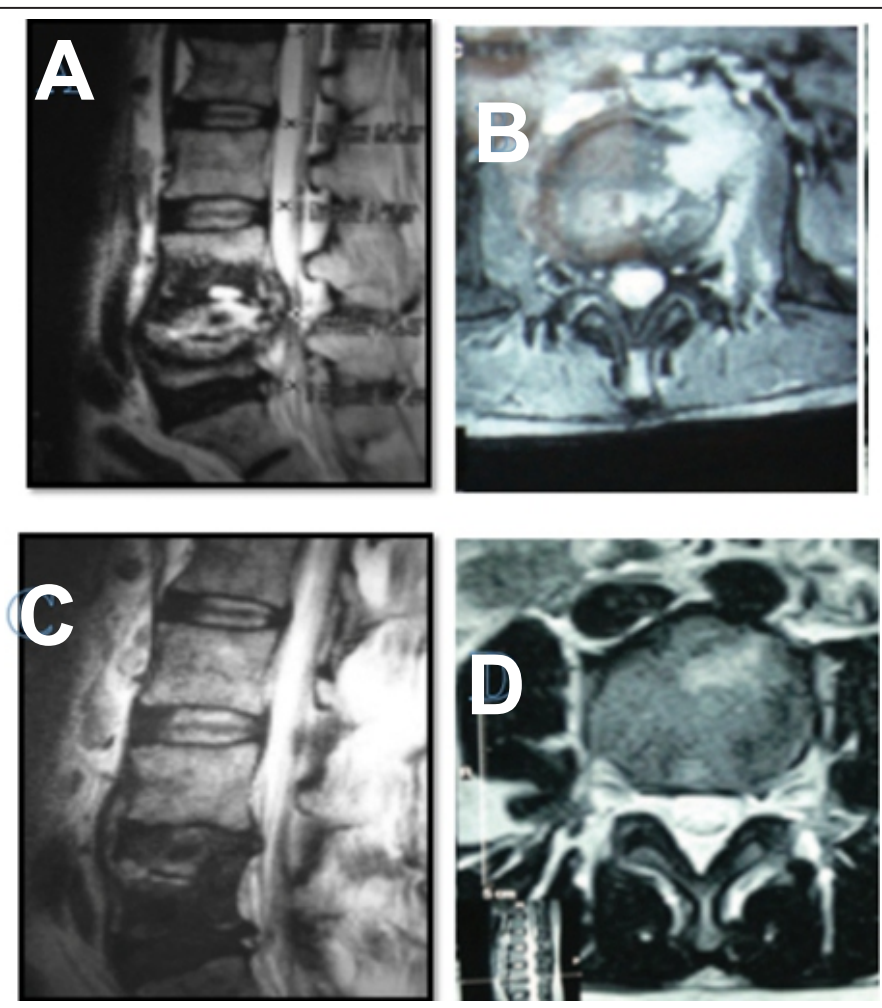

Figure 1: 66 years old female patients with spinal tuberculosis. (A \& B) T2 weighted images of MRI shows L4-L5 tubercular spondylodiscitis, paradiscal involvement with compression over the thecal sac. (C \& D) T2 images of MRI after 12 months of ATT shows recovery from infection with fatty marrow changes.

India, high-density population, urbanization with poverty, large scale migration, the emergence of HIV and drug resistant tuberculosis has caused a high burden of pulmonary tuberculosis, and the incidence of spinal tuberculosis is expected to be proportionately high [3]. Spinal tuberculosis generally presents with nonspecific back pain in the early stages. The plain radiograph changes lag behind the clinical presentation by $8-12$ weeks and it leads to a difficult diagnosis. Magnetic renaissance imaging (MRI) is extremely sensitive for the detection of bone edema [5]. Sometimes, there are no clinico-radiological studies that can diagnose spinal tuberculosis certainty and it makes diagnosis difficult. The diagnosis is based on the isolation of mycobacteria on culture tissue obtained by performing a spinal biopsy. The CT-guided biopsy and ATT as per drug sensitivity of mycobacteria have shown encouraging results [6]. Indication for surgical management of spinal tuberculosis includes severe or progressive neurological deficit or severe vertebral destruction with the potential for significant kyphosis. Other indications for surgical treatment include uncertain diagnosis, a potentially unstable spinal segment with needs stabilization and refractory disease [7]. The current philosophy for the nonoperative treatment of spinal tuberculosis to prevent permanent neurological disability and minimize spinal deformity includes
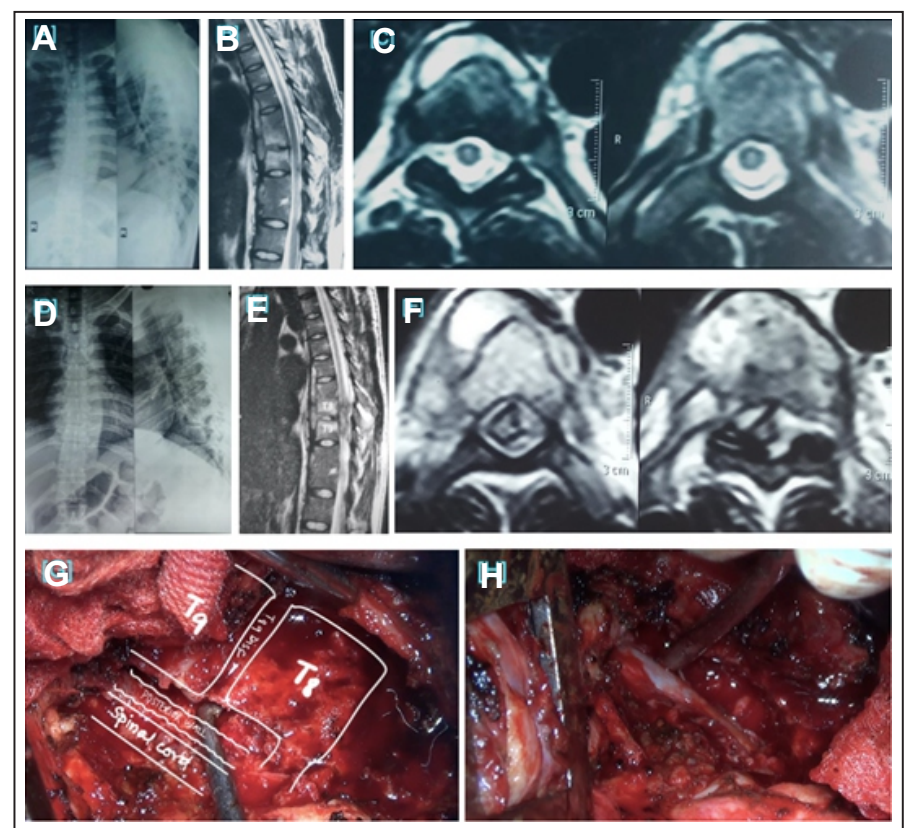

Figure 2: 36 year-old-male patients with dorsal spine tuberculosis. (A)Radiograph of dorsolumbar spine shows local kyphosis at T8-T9 level with decreased disc space with T10-T11 block vertebrae. (B,C) T2 weighted images of MRI shows T8-T9 tubercular spondylodiscitis with a prevertebral collection without cord compression. (D) 3-month post biopsy patient present with paraparesis. (E,F) MRI shows significant cord compression with epidural abscess extending from $\mathrm{T} 6$ to $\mathrm{T} 11 .(\mathrm{G}, \mathrm{H})$ The patient was operated with thoracotomy, decompression and debridement.

early detection, confirmation of diagnosis, indications for nonoperative treatment, appropriate ATT, periodic evaluation, activity restriction and supportive bracing, stopping ATT after confirming disease healing and follow-up post-treatment for possible relapses $[8,24]$. Uncomplicated spinal tuberculosis is a medical disease but there is an increasing trend toward more and more surgical management of thoracolumbar spinal tuberculosis since the last decade [24]. Our is a tertiary care public institute of western India which is situated in one of the most densely populated cities of the world. Therefore, our institute has a high tuberculosis patient load. In this prospective study, we present our experience of conservative treated thoracolumbar spine tuberculosis. A study objective was to assess the clinical and radiological outcomes of the conservative management of thoracolumbar spine tuberculosis and reiterate the importance of conservative management.

\section{Materials and Methods}

After approval from the institutional ethics committee, this prospective study was conducted from May 2016 to April 2019 at a single institute. Informed consent was taken from all the patients. The diagnosis of spinal tuberculosis was based on cliniçal and neneurological examination with imaging ing features. 
Spinal tuberculosis was considered when a patient presented with the characteristic clinical features of pain, constitutional symptoms, localized tenderness, deformity and the essential radiological criteria were the destruction of two or more contiguous vertebrae and opposed endplates, disc infection, along with a paraspinal mass or abscess. Suspected MRI lesions were those which showed an isointense lesion on $\mathrm{T} 1$ or a high signal lesion on T2 involving the disc, body, paravertebral region and sometimes extending into the epidural space or psoas fascia (Fig. 1).

The inclusion criteria were: (a) Biopsy proven thoracolumbar spinal tuberculosis, (b) Age 20 to 50 years, (c) Kumar's Clinico-radiological classification: Stage-I, II and III, (d) Neurological deficit (ASIA scale C, D), and (e) Jain modified grading systems for Evaluation of paraplegia in tuberculosis of the spine: Stage I and II. The exclusion criteria were: (a) atypical imaging features like single vertebral involvement, (b) isolated involvement of the posterior spinal segment, (c) noncontiguous vertebral involvement, (d) previously operated for spine pathology, (e) patients on ATT or previous ATT taken, (f) immunocompromised patients, (g) other significant comorbidities, and (h) frank instability on radiographs.

\section{Pre-treatment workup and Assessment}

Clinical and functional assessment: (a) Demographic data: age, sex, duration of a presentation and duration of weakness, (b) pain score- Visual Analog Scale- VAS, (c) Oswestry Disability Index- ODI, (d) neurological- ASIA impairment scale, and (e) clinico-radiological staging- Kumar's criteria. A laboratory assessment: Complete blood counts, Erythrocyte sedimentation rate (ESR), CRP, blood sugar, liver and renal function test. A radiological assessment: Chest and spine radiographs, computerized tomography (CT) scan, Magnetic resonance imaging (MRI).

Treatment Protocol: All patients were initially subject to CTguided biopsy for the confirmation of diagnosis. The biopsy was performed using CT-guidance, for which patients were placed in a prone or lateral position on the CT scanner table. Few pilot scans were taken beforehand to tailor the approach for safe insertion of the spinal needle and localization of the lesion. An 18-gauge spinal needle was inserted percutaneously, depth and route of which were dictated by CT scan. Necrotic material was aspirated as much as possible using a $10 \mathrm{ml}$ syringe. The sample collected from the biopsy was sent for acid-fast bacillus (AFB) smear, GeneXpert and culture and sensitivity of Mycobacterium tuberculosis. After the GeneXpert report Anti-tuberculosis therapy (ATT) was started in all patients as per chest physician and Revised National Tuberculosis Control Program (RNTCP guidelines Government of India) guidelines [9]. All patients received
Conservative treatment in the form of Fixed-dose combination-FDC ATT for a minimum of 12 months. Pyridoxine was also added to the regimen prophylactically for three months. Calcium and high protein diet, Vitamin D3 60000 IU oral tablet once a week for 4 weeks given as a supplement. All the patients prescribed an appropriate brace or collar for 6 months. During the follow-up visits, patients were managed and monitored for pain, ESR, CRP and neurological status.

Follow-up was done initially monthly for the first 3 months, then at 6-months and last follow-up at 12-months. The spinal radiographs were taken in every follow-up. MRI was done at the final follow-up in affording patients only.

The endpoint of ATT was: (a) Clinical- Improvement of constitution symptoms, pain relief, improved spinal movements, (b) Radiographs well-defined endplates, improvement in bone rarefaction, sclerosis, bony fusion, and (c) MRI- Loss of high signal, fatty changes in vertebra on T2 weighted MRI along with resorption of abscess further confirmed the resolution of infection.

Surgical intervention was done in patients with failed repeated biopsy, persistent neurology after the treatment or worsening of neurology (Fig.3).

Statistical Analysis: The data analysed Using SPSS 20.0 software (SPSS, Inc., Chicago, IL), a paired sample t-test was used to compare the pre and post-treatment clinical and radiographic data, with a $\mathrm{P}$-value of 0.05 considered to be statistically significant and results are given in percentages. The Chi-square test was used to find out the association between

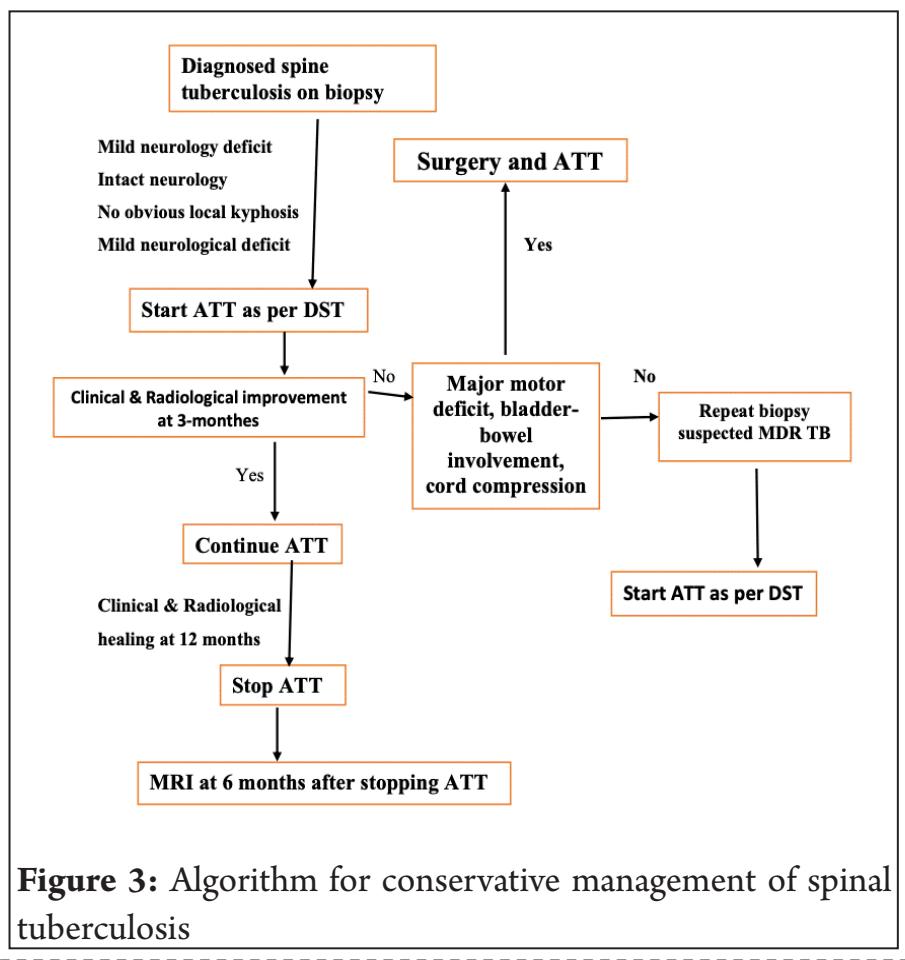


two dichotomous variables. The relative risk with its $95 \%$ confidence interval was calculated. Multivariate logistic regression analysis was performed to find out significant factors associated with the outcome. Clinical variables included in the statistical analysis were age, sex, duration of symptom, neurological deficient concomitant tuberculosis history. Imaging parameters included a number of vertebrae involved, disc space reduction, loss of vertebral height, paravertebral collection, cord signal changes, and spinal extension of an abscess.

\section{Results}

188 patients (Male 56, Female 132) were included in the study. The mean age of individuals was $33.9 \pm 13.7$ years $(20-50$ years). Presenting complaints included pain in 180 (95.7\%), Neurological deficit ASIA-C 160 and ASIA-D 28 patients. An MRI of the spine showed a paravertebral abscess in 148 (78.7\%), Vertebral height Loss in 55 (29.25\%) and disc destruction in 157 (83.51\%) patients and paravertebral collection in all patients $100 \%$, whereas, psoas abscess was seen in $16(8.5 \%)$ patients. The D6-D7 level was involved in 13 (6.91\%) patients, 13 (6.91\%) had D7-D8 level, 18 (9.57\%) had D8-D9 level, 19 (10.10\%) had D10-D11 level, 41 (21.80\%) had D11-D12 level, 37 (19.68\%) had D12-L1, 21 (11.17\%) had L1-L2, 17 (9.04\%) had L2-L3, 5 (2.65\%) had L3-L4 and 4 (2.12\%) had L4-L5. According to Jain's modified grading system for evaluation of paraplegia classification 157 patients had in stage-I (83.51\%) and 31 patients had stage-II (16.48\%). Based on of Kumar's clinico-radiological classification 71 patients were stage-I (37.76\%), 59 (31.38) stage-II and 58 (30.85) stage-II (Table 1).

All patients were initially planned for biopsy; biopsy has successfully performed on 170 (90.42\%) patients while it failed in 18 (9.57\%) patients. All patients underwent a single aspiration except 8 required repeat biopsy. The total amount of material aspirated during a successful biopsy varied from $1 \mathrm{ml}$ to as much as $200 \mathrm{ml}$ with a mean of $15.2 \mathrm{ml}$. Following a successful biopsy, the AFB smear was positive in 152 (89.7\%) patients. Mycobacterium bacteria were isolated in 159 patients (93.52\%) and failed to isolate in 11 patients $(6.47 \%)$ in culture media. GeneXpert was positive in 150 patients (88.23), Rifampicin resistance detected in 18 patients (9.57) and negative in 2-patients (1.17). Histopathology report suggested mycobacterium in 166 patients $(97.64 \%)$ and inconclusive finding in 4 patients $(2.35 \%)$ (Table 2$)$.

The mean ESR during the presentation was $67.2014 .80 \mathrm{~mm}$ in the first hour, which fell to $35.35 \pm 3.83 \mathrm{~mm}$ in the first hour at 1 month on ATT. At the end of treatment, the mean ESR was $16.24 \pm 2.50 \mathrm{~mm}$. The mean CRP during the presentation was $54.1326 .96(\mathrm{mg} / \mathrm{dl})$, which fell to $38.46116 .53 \mathrm{mg} / \mathrm{dl}$ at 1 month on ATT. At the end of treatment, mean CRP was 6.05
$5.01 \mathrm{mg} / \mathrm{dl}$, which corresponded with a resolution of symptoms (Table 3 ).

Out of 170 biopsy successful patients, 142 were ASIA-C and 28 ASIA-D. 152 completely recovered ASIA-E. Eleven Patients remain the same with neurology. Seven patients had to worsen in neurology. These all 18 patients managed by surgically and repeat biopsy sample 17 came positive to MDR tuberculosis and that might be a cause of failed conservative treatment, in

\begin{tabular}{|c|c|c|}
\hline Characteristics & Number & Percentage \\
\hline \multicolumn{3}{|c|}{ Gender } \\
\hline Male & 56 & 29.78 \\
\hline Female & 132 & 70.21 \\
\hline \multicolumn{3}{|c|}{ Clinical features } \\
\hline Backache & 188 & 100 \\
\hline Local Tenderness & 180 & 95.74 \\
\hline Fever & 70 & 37.23 \\
\hline Kyphosis & 55 & 29.25 \\
\hline Weight Loss & 40 & 21.27 \\
\hline \multicolumn{3}{|c|}{ Level of spinal involvement } \\
\hline D6-D7 & 13 & 6.91 \\
\hline D7-D8 & 13 & 6.91 \\
\hline D9-10 & 18 & 9.57 \\
\hline D10-D11 & 19 & 10.1 \\
\hline D11-D12 & 41 & 21.8 \\
\hline D12-L1 & 37 & 19.68 \\
\hline L1-L2 & 21 & 11.17 \\
\hline L2-L3 & 17 & 9.04 \\
\hline L3-L4 & 5 & 2.65 \\
\hline L4-L5 & 4 & 2.12 \\
\hline Total & 188 & 100 \\
\hline \multicolumn{3}{|c|}{ Severity of bone destruction by Plain radiograph } \\
\hline Wedging & 140 & 74.46 \\
\hline Compression Fracture & 30 & 15.95 \\
\hline Both & 18 & 9.57 \\
\hline \multicolumn{3}{|c|}{ Kumar's Clinico-radiological classification } \\
\hline Stage I & 71 & 37.76 \\
\hline Stage II & 59 & 31.38 \\
\hline Stage III & 58 & 30.85 \\
\hline Total & 188 & 100 \\
\hline \multicolumn{3}{|c|}{$\begin{array}{l}\text { Jain modified grading systems for Evaluation of } \\
\text { paraplegia in tuberculosis of the spine }\end{array}$} \\
\hline Stage I & 157 & 83.51 \\
\hline Stage II & 31 & 16.48 \\
\hline Total & 188 & 100 \\
\hline
\end{tabular}




\begin{tabular}{|c|c|c||}
\hline \multicolumn{3}{|c|}{ Table 2 Microbiology and Pathology result } \\
\hline \multicolumn{3}{|c|}{ CT guided biopsy $(\mathbf{n}=\mathbf{1 8 8})$} \\
\hline Successful & 170 & 90.42 \\
\hline Failed & 18 & 9.57 \\
\hline AFB smear (n=170) & \\
\hline Positive & 152 & 89.41 \\
\hline Negative & 18 & 9.57 \\
\hline GeneXpert (n=170) & \\
\hline Sensitive & 150 & 88.23 \\
\hline Resistance to Rif & 18 & 9.57 \\
\hline Negative & 2 & 1.17 \\
\hline TB culture (n=170) & & \\
\hline MTB isolated & 159 & 93.52 \\
\hline Failed & 11 & 6.47 \\
\hline Histopathology (n=170) & \\
\hline Mycobacterium Positive & 166 & 97.64 \\
\hline Inconclusive finding & 4 & 2.35 \\
\hline
\end{tabular}

Table 3: The improvement status of cases on the basis of ESR \& CRP changes

\begin{tabular}{||c|c|c|c|c||}
\hline & \multicolumn{2}{|c|}{ ESR } & \multicolumn{2}{c|}{ CRP (mg/dl) } \\
& Mean & p-value & Mean & p-value \\
Pre-treatment & $67.2 \pm 14.8$ & - & $54.13 \pm 26.96$ & - \\
\hline First Visit & $35.35 \pm 3.83$ & $<0.005$ & $38.46 \pm 116.53$ & $<0.005$ \\
\hline Final Visit & $16.24 \pm 2.50$ & $<0.005$ & $6.05 \pm 5.01$ & $<0.005$ \\
\hline
\end{tabular}

Table 4 Pre- and post-treatment neurological status using ASIS scale $(\mathrm{n}=170)$

\begin{tabular}{|c|c|c|c|c|c|c||}
\hline Pre-treatment & $\begin{array}{c}\text { No of } \\
\text { patients }\end{array}$ & A & B & C & D & E \\
\hline ASIA C & 142 & & 3 & 6 & & 133 \\
ASIA D & 28 & & & 4 & 5 & 19 \\
\hline
\end{tabular}

\begin{tabular}{|c|c|c|c|}
\hline \multicolumn{4}{|c|}{ Table 5: Functional and radiological results } \\
\hline & Mean & SD & p value \\
\hline \multicolumn{4}{|c|}{ VAS } \\
\hline Preoperative & 7.9 & 0.6 & $<0.05$ \\
\hline Postoperative 1 month & 4 & 0.54 & \\
\hline Final follow-up & 2.9 & 0.54 & \\
\hline \multicolumn{4}{|c|}{ ODI } \\
\hline Preoperative & 77.1 & 6.9 & $<0.05$ \\
\hline Postoperative 1 month & 30.5 & 6.5 & \\
\hline Final follow-up & 21.3 & 6.7 & \\
\hline \multicolumn{4}{|c|}{ Improvement of kyphotic angle on Radiograph (Degree) } \\
\hline Preoperative & 5.68 & 3.84 & $<0.005$ \\
\hline Post treatment & 6.51 & 3.88 & \\
\hline \multicolumn{4}{|c|}{$\begin{array}{l}\text { VAS: Visual Analog Score; ODI: Oswestry Disability Index; SD } \\
\text { Standard deviation }\end{array}$} \\
\hline
\end{tabular}

Table 6. Surgical Detail of failed cases

\begin{tabular}{|l|c|c|}
\hline Procedure & $\begin{array}{c}\text { Failed } \\
\text { Biopsy }\end{array}$ & $\begin{array}{c}\text { Successful } \\
\text { biopsy }\end{array}$ \\
\hline
\end{tabular}

Thoracotomy and decompression Dorsal laminectomy and decompression Thoracoscopic-assisted anterior debridement. iliac bone autograft and instrumentation Thoracotomy, iliac bone autograft and instrumentation

$1-1$

21

20

Costotransversectomy decompression, cage and pedicle screw fixation

Dorsal Transforaminal debridement, interbody fusion and posterior instrumentation. Lumbar- transforaminal debridement, interbody fusion and posterior instrumentation. Retroperitoneal decompression, cage and instrumentation. Total

1

5

$5 \quad 7$

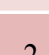

2

3 2

\begin{tabular}{|c|c|c|}
\hline and instrumentation. & 2 & 2 \\
\hline Total & 18 & 18 \\
\hline
\end{tabular}

\section{Table 7. Complication $(\mathrm{n}=1 \mathbf{1 0})$}

\begin{tabular}{|c|c|c|}
\hline MDR Positive & 17 & 10 \\
\hline No Neurological Improvement & 11 & 6.47 \\
\hline Worsening of neurology & 7 & 4.11 \\
\hline Surgery & 18 & 10.58 \\
\hline
\end{tabular}

one patient failure of conservative treatment cause was unknown. A second line ATT was given in these 17 patients as per pulmonologist advice (Table 4).

VAS was significantly improved from mean value preoperative $7.90 \pm 0.60$ to $4.0 \pm 0.54$ postoperative 1 month and $2.90 \pm 0.54$ at the final follow-up. ODI was improved from mean value preoperative $77.10 \pm 6.90$ to $30.50 \pm 6.50$ postoperative 1 month and $21.30 \pm 6.70$ at final follow-up. Pre-treatment mean kyphosis was $5.68 \pm 3.84$ that improved to $6.51 \pm 3.88$ posttreatment. The highly significant changes (increase) were found in the kyphotic angle $(\mathrm{p}<0.005)$ (Table 5).

Decompressive and or/with instrumentation surgery was performed in 36 patients (Table 6). Out of these surgical candidates, 18 had failed biopsy attempts and 18 had indications despite successful biopsy including persistent neurological deficit or worsening of neurology due to mechanical compression-kyphosis. 152 (80.85\%) patients managed completely conservative at the end of our study. 141 (92.76\%) in our study showed good compliance with ATT and fully recovered without any residual weakness or pain. However, 11 (7.23\%) patients were non-compliant with the treatment. Patients who demonstrated poor compliance deteriorated during the course and required surgery. Two compliant patients died of an unrelated medical condition before the completion of the regimen. Moreover, none of the patients showed any signs of the recurrence of $\mathrm{TB}$, in the spine or any other region of the body, during the follow-up period (Table 7).

16 | Back Bone: The Spine Journal | Volume 1 | Issue 1 | April-September 2021 | Page 12-18 


\section{Discussion}

The thoracolumbar spine is the most common region within the spine to be affected with tuberculosis. Due to inherent stability offered by the rib cage, tuberculosis of the thoracic spine often presents late with an established kyphosis and neurological deficit, although most patients with early disease are treated successfully nonoperatively. Spinal tuberculosis infection responds extremely well to ATT based on the drug susceptibility pattern of the mycobacteria. The average age at presentation was $33.90 \pm 3.50$ years ( 20 to 50 years). The total number of patients was 188 and the male-female ratio was 1:2.3. This ratio was opposite to other all studies [10]. The most common symptom was back pain in patients in our study. Axial pain of insidious onset is regarded as one of the most common symptoms of spinal tuberculosis. However, it can present itself with a wide range of symptoms and complications [11-12]. The diagnosis can be made through clinical, radiological and microbiological studies. Radiological studies, especially MRI play a key role in establishing the diagnosis of spinal Tuberculosis[13]. The difference between inflammatory and neoplastic pathologies may not be appreciated on plain radiographs or CT scans. Hence, MRI is needed which reveals tuberculosis as a contrast-enhancing rim on T2-weighted images [14]. This appearance can be readily differentiated from a metastatic lesion first involving the pedicles, which are initially spared in spinal tuberculosis. The CT-guided biopsy and culture of mycobacteria is the most efficacious method for the establishment of a confirmed diagnosis of spinal tuberculosis [15]. An adequate response to ATT can also be used to establish the diagnosis, recommended observation period for drug response is 3 to 4 weeks in patients with neurological deficits and 6 to 8 weeks in those with intact neurology [16]. However, labeling the patient as a nonresponder based on a subjective assessment period is not recommended and remains a matter of debate. The key to successful conservative treatment is early detection of the infection, followed by microbiological confirmation and identification of drug resistance. The AFB smear was prepared using the Ziehl-Neelsen stain and Lowenstein-Jensen medium was used for the culture of the organism. Newer novel liquid culture media enables rapid growth of the mycobacteria within 10 to 14 days and tests such as line probe assays and GeneXpert enable the identification of drug resistance rapidly [15]. Most of the studies observed that the thoracic spine was the most common site of involvement followed by the lumbar, the thoracolumbar, and the cervical spine [17-18]. Our study had also found a similar result for the common site of spinal tuberculosis. Primary drug resistance is on the rise. Hence, it is vital to obtain a biopsy before starting ATT. The success rate of CT-biopsy was $90.6 \%$ in our study and sufficient sample material collected for AFB, GeneXpert and culture. Some studies reported that samples from CT-guided biopsy were often inadequate [19]. The CT-guided biopsy can drain paraspinal collection that reduces damage to adjacent structures and also eliminates the risk of surgery and general anaesthesia related complications $[22,23]$. We had a positive AFB smear in $89.7 \%$ and a positive culture in all successful biopsy. However, a positive culture of $76 \%$ has been reported by staatz et al using CT-guided biopsy [20, 21]. A multidrug ATT inappropriate dosage as per patient body weight is compulsory to reduce the risk of acquired drug resistance. The goal is to rapidly reduce bacterial load during the intensive phase and reduce the risk of relapse in the continuation phase [23]. A highly significant reduction in ESR was found at each follow-up relative to before treatment $(p<0.005)$. A highly significant reduction in CRP was found after the first visit, second visit and final follow-up ( $p<0.005$ for each visit). The VAS for back pain was significantly improved from the mean value preoperative $7.90 \pm 0.60$ to $4.0 \pm 0.54$ postoperative one month and $2.90 \pm 0.54$ at the final follow-up. The ODI was improved from mean value preoperative $77.10 \pm 6.90$ to 30.50 \pm 6.50 postoperative one month and $21.30 \pm 6.70$ at the final follow-up. In our study, pre-treatment mean kyphosis was 5.68 \pm 3.84 that improved to $6.51 \pm 3.88$ post-treatment that was significant $(\mathrm{p}<0.005)$. Spinal tuberculosis patients with mild neurological deficit, destruction of less than three-fourths of a vertebral body, no evidence of mobile instability and kyphosis less than 30 degree can be considered for conservative treatment $[7,17]$. Out of 170 biopsy successful patients, pretreatment 142 patients had ASIA-C and 28 patients had ASIAD. Post-treatment, 152 patients completely recovered with ASIA-E. Post-treatment, eleven patients remained the same in neurology and seven patients had worsened neurology. On repeat biopsy of these 18 patients, 17 came positive for MDR tuberculosis and that is a chief cause of treatment failure. These 18-patients required some type of decompression and debridement surgery (Fig. 2). Management of MDR tuberculosis is a very difficult task, as the second line ATT is less effective and more costly and the duration of treatment is longer. It is very important to identify the risk factors for failure of conservative treatment with first-line ATT [24]. In our study 152 patients, $89.41 \%$ out of 170 successful biopsies demonstrated good compliance with the treatment regimen and showed complete recovery at the end of treatment. Reasons for failure of conservative treatment are (a) Inadequate diagnosis, (b) Inappropriate patient section, (c) acquired drug resistance and (d) non-compliance patients. Drawbacks of this study are the lack of a comparative study group and short follow-up. Studies with the larger patient number and longer follow-up are required for confirmation of our results. Prolonged post-treatment follow-up is essential to detect relapse. 


\section{Conclusions}

Early diagnosis and early treatment of spinal tuberculosis before the development of spinal deformity seem to be essential for better outcomes. Uncomplicated biopsy proven spine TB responds well to nonoperative management. However, the correct regimen, correct dosage and correct duration of ATT are vital for spinal tuberculosis treatment. Periodic evaluation is a must to look for evidence of improvement and the adverse effects of ATT.

\section{References}

1. Tuli SM. Historical aspects of pott's disease (spinal tuberculosis) management. Eur spine J2013;22 (suppl4):529-53

2.Dobson J.Percivall Pott. Ann R Coll Surg Eng 1972;50(1):54-65

3. Gautam MP, Karki P, Rijal S, Singh R. Pott's spine and Pott's paraplegia. J Nep MedAssoc2005;44(159):106-15

4. Global tuberculosis report 2017: World Health Organization. Available from apps.who.int/iris/bitstream/hamdle/10665/259366/9789241565516eng.pdf

5. Moorthy S,Prabhu NK. Spectrum of MR imaging findings in spinal tuberculosis. AIRAm J Roentgenol 2002; 179(4): 979-983

6. Oguz E, Sehirlioglu A, Altinmakas $M$, et al. A new classification and guide for surgical treatment of spinal tuberculosis. Int Orthop 2008;32:127-33.

7. Jain AK. Tuberculosis of the spine: A fresh look at an old diseas. J Bone Joint surgery Br 2010:92 (7): 905-913

8. Patil SS, Mohite S, Varna R, Bhojraj SY, Nene AM. Non-surgical management of cord compression in tuberculosis: a series of surprises. Asian Spine J 2014:8(3): 315-321

9. N'Dri Oka D, N'Dri-Yoboue MA, Varlet G, Haidara A, Ba Zeze V. Spinal tuberculosis. Epidemiologic and diagnostic aspects: a study of 28 clinical observations. Sante 2004;14:81-4.

10. Gasbarrini AL, Bertoldi E, Mazzetti M, et al. Clinical features, diagnostic and therapeutic approaches to haematogenous vertebral osteomyelitis. Eur Rev Med Pharmacol Sci2005;9:53-66.

11. Kumar R. Spinal tuberculosis: with reference to the children of northern India. Childs Nerv Syst 2005;21:19-26.

12. Forrester DM. Infectious spondylitis. Semin Ultra- sound CT MR 2004;25:46173.

13. Umredkar A, Mohindra S, Chhabra R, Gupta R. Ver-tebral body hyperostosis as a presentation of Pott's disease: a report of two cases and literature review. Neurol
India 2010;58:125-7.

14. Boachie-Adjei O, Squillante RG. Tuberculosis of the spine. Orthop Clin North Am 1996;27:95-103.

15. Rajasekaran S, Khandelwal G. Drug therapy in spinal tuberculosis. Eur Spine J. 2013; 22(4):587-93.

16. Tuli SM, Kumar K, Sen PC, Penetration of anti-tubercular drugs in clinical osteoarticular tubercular lesions. Acta Orthoop Scand 1977: 48(4):362-368

17. Ravindra Kumar Garg,Dilip Singh Somvanshi, et al. Spinal tuberculosis: A review.JSpinal Cord Med.2011 Sep; 34(5): 440-454.

18. Dinc H, Ahmetoglu A, Baykal S, Sari A, Sayil O, Gumele HR. Image-guided percutaneous drainage of tuberculous iliopsoas and spondylodiskitic abscesses: midterm results. Radiology 2002;225:353-8.

19. Staatz G, Adam GB, Keulers P, Vorwerk D, Gunther RW. Spondylodiskitic abscesses: CT-guided percutaneous catheter drainage. Radiology 1998;208:363-7.

20. Zhang XF, Wang Y, Liu ZS, et al. Percutaneous focal debridement and local chemotherapy in the treatment of spinal tuberculous abscesses (Chin). Chin J Spine Cord 2005; 15:528-30.

21. Muzii VF, Mariottini A, Zalaffi A, Carangelo BR, Palma L. Cervical spine epidural abscess: experience with microsurgical treatment in eight cases. $J$ Neurosurg Spine 2006; 5:392-7.

22. Rajasekaran S, Prasad Shetty A, Dheenadhayalan J, Shashidhar Reddy J, Naresh-Babu J, Kishen T. Morphological changes during growth in healed childhood spinal tuberculosis: a 15-year prospective study of 61 children treated with ambulatory chemotherapy. JPediatr Orthop 2006;26:716-24.

23. Dunn R. The medical management of spinal tuberculosis. SA Orthopaedic Journal 2010: 9(1):37-41

24. Nene A, Bhojraj S. Result of nonsurgical treatment of thoracic spine tuberculosis in adults. Spine J2005;5(1)79-84
Conflict of Interest: NIL Source of Support: NIL

\section{How to Cite this Article}

Kakadiya G, Soni Y, Saindane K, Gohil K, Chaudhary K, Shakya A | Conservative Management of Thoracolumbar Spinal Tuberculosis in The Tertiary Care Hospital of India | Back Bone: The Spine Journal | April-September 2021;2(1):12-18. 